\title{
FOCAL ADHESION QUANTIFICATION - A NEW ASSAY OF MATERIAL BIOCOMPATIBILITY? : REVIEW
}

\author{
Owen, G Rh ${ }^{*}$, Meredith, DO ${ }^{2}$, ap Gwynn, ${ }^{3}$ and Richards, $\mathrm{RG}^{2}$ \\ ${ }^{1}$ New York Structural Biology Centre, New York 10027, USA. \\ ${ }^{2} \mathrm{AO}$ Research Institute, Davos, Switzerland. \\ ${ }^{3}$ Institute of Biological Sciences, The University of Wales, Aberystwyth, Wales, UK.
}

\begin{abstract}
The development of novel synthetic biomaterials is necessitated by the increasing demand for accelerated healing of tissues following surgical intervention. Strict testing of such materials is necessary before application. Currently, before any material can be marketed, approval by regulatory organisations such as the FDA is required. Presently, in vitro testing is performed as a prerequisite to in vivo evaluation. The in vitro techniques currently employed do not reflect the progress in our understanding of extra and intra-cellular processes, with far more sensitive in vitro evaluations now available. Obtaining quantifiable data is increasingly relevant to evaluating events occurring in vivo. Quantifying cell adhesion to surfaces provides some of this data as an initial assessment method. Major developments in this field are occurring but many investigators still use less than optimal methods for assessing biomaterials. The relevance of using cell adhesion assays to help determine biomaterial biocompatibility is reviewed. Additionally, current in vitro methods of evaluating biomaterials are discussed in the context of novel testing concepts developed by the authors.
\end{abstract}

Key Words: Review, Cell adhesion, Biocompatibility testing, Biomaterials, Cytocompatibility.

\footnotetext{
*Address for Correspondence:

G, Rh. Owen,

New York Structural Biology Center,

New York 10027,

USA.
}

Telephone number: 212-939-0660 ext 322

E-mail: gowen@nysbc.org

\section{Introduction}

The goal of this review is to provide the reader with an overview of the concepts in cytocompatibility testing with particular emphasis on novel techniques developed by the authors. As this is not a comprehensive review of past methods, we have attempted to include sufficient references so that the reader can be directed towards the relevant publications. A brief introduction to the focal adhesion will be given including a detailed description of protein organisation and their role in signal transduction. The review will then focus on methods that have been used to quantify cell adhesion and discussing the approaches that may be employed to improve the sensitivity of the method. This will lead to an explanation of the new method to predict the cyto and biocompatibility of materials and surface topography by quantifying the focal adhesion area compared to the total cell area. The advantages and disadvantages of the method will then be discussed followed by an examination of the potential for future methodology development derived from the technique.

For evaluation of a material intended for use as an implantable device, it is necessary to understand the processes that occur as a consequence of the implantation procedure. Implantation of materials results in the formation of a wound and the ensuing wound healing cascade is the body's natural response, in which damaged tissue is regenerated by processes of coagulation, inflammation and eventual repair as well as a tissue remodelling phase. Damaged tissue is regenerated by the migration of cells to the injured area, induced by the release of chemoattractant signals from the damaged tissue. Tissue healing is eventually accomplished by a combination of cell proliferation and cell matrix synthesis to form the regenerated tissue. Anderson (1993) and Dee et al. (2002), provide a more comprehensive explanation of the process.

When implant materials are placed in the body, cells migrate to the site from adjoining tissues. Once this has occured, the cells' perception of the implant determines the body's reaction for tolerance and acceptance of the foreign object. The cells do not make contact directly with the actual surface, but to extracellular signals such as proteins that have adsorbed immediately to the surface from the blood (Baier and Dutton, 1969; Wilson et al., 2005) and interstitial fluids. It is these extracellular cues that are translated into cellular responses by the nucleus, to the areas of cell material adhesion. These sites, known 


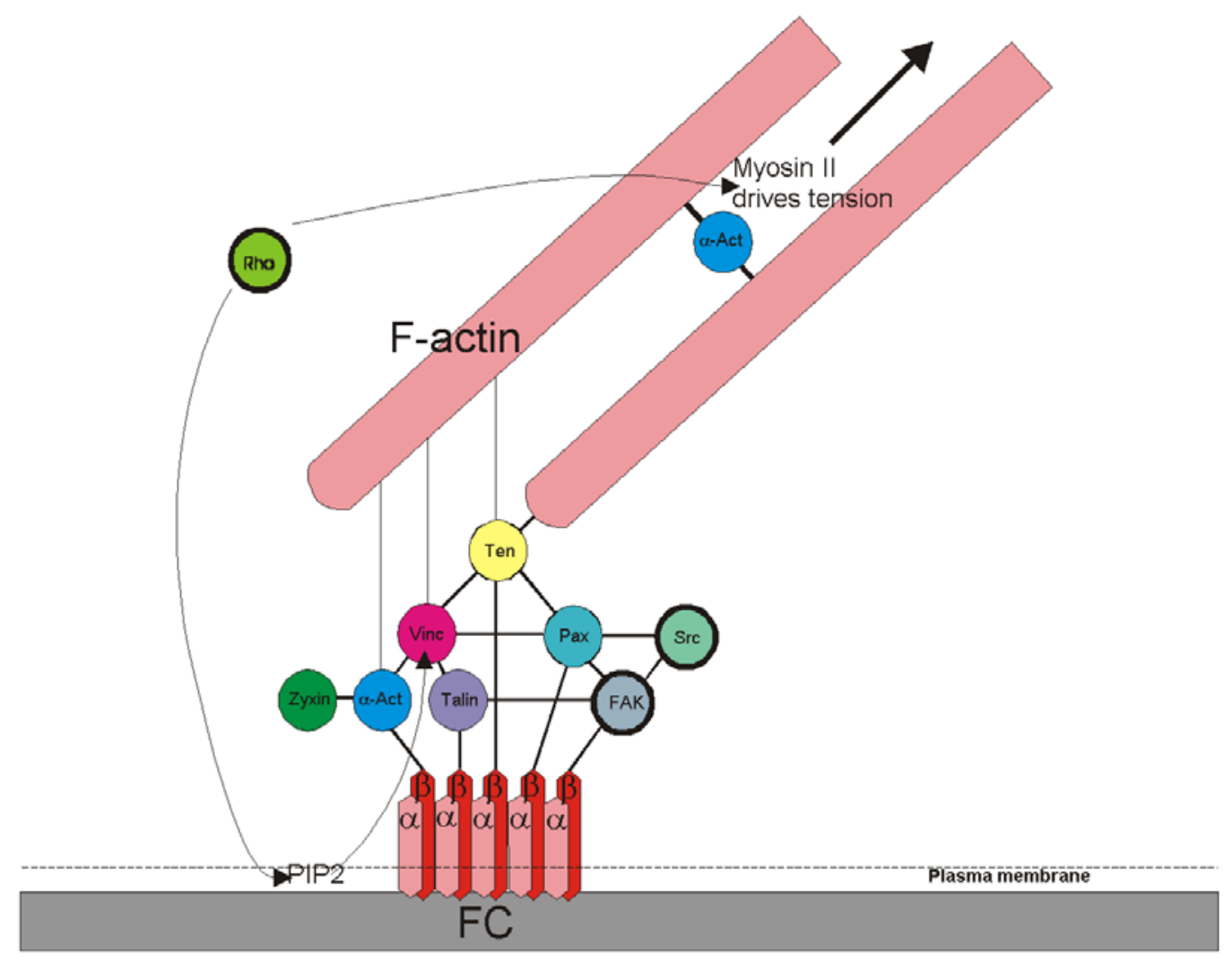

Figure 1. Diagrammatic representation of the spatial interaction of most of the focal adhesion linker and signalling proteins. Abbreviations $\alpha$-act $=\alpha$-actinin, $\mathrm{Pax}=$ paxillin, Vinc $=$ vinculin, $\mathrm{Ten}=$ tensin, $\mathrm{FAK}=$ focal adhesion kinase, PIP2 = phosphotidyl inositol-4-5 bisphosphate, $\alpha \& \beta=$ integrins, FC $=$ focal contact. Modified from "Guidebook to the extracellular matrix, anchor and adhesion proteins". 2nd edition. Editors T Kreis and R.Vale. Oxford University Press, UK. pp. 5.

as focal contacts, focal adhesion plaques or focal adhesions (Ambrose, 1961; Curtis, 1964; Cornell, 1969; Izzard and Lochner, 1976), are where the transmembrane proteins are attached closely to the extra cellular matrix (ECM) (Burridge et al., 1988). They are connected internally with the actin microfilament component of the cytoskeleton (Figure 1).

\section{The Focal Adhesion}

Focal adhesions were first observed to form between cells and solids by Ambrose (1961) using the surface contact light microscope and later Curtis (1964) reported that their distance of closest approach was found to be approximately $10 \mathrm{~nm}$, using interference reflection microscopy (IRM). Two morphological variants of the focal adhesion exist; the 'dot' and 'dash' variants (Bershadsky et al., 1985). 'Dot', or small initial, contacts are constituted of transmembrane and some linker proteins but are not associated with actin bundles. These are the predominant contact type, with dimensions of $0.2-0.5 \mu \mathrm{m}$, and are mainly located at the active edge of the cell. Elongated large mature, or 'dash', contacts are also composed of transmembrane and linker proteins but differ due to their association with the cytoskeletal actin bundles. Dash contacts are $2-10 \mu \mathrm{m}$ in length and $0.5 \mu \mathrm{m}$ in width and are located centrally in parts of the lamellae, areas of the endoplasm and under the nucleus. Initial formation of 'dot' contacts occurs followed by maturation into 'dash' contacts where the association with actin bundles occurs (Cooper, 1987). Both types of contact are important in cell adhesion since both can transmit force (Dembo and Wang, 1999). The importance of the force bearing capabilities and the transmembrane location of the focal adhesion will become apparent later in this review.

The focal adhesion complex is composed of a high density of proteins that attach the extracellular portion of the cell to the intracellular cytoskeletal portion. Transmembrane proteins, known as integrins (Hynes, 1987; 2002), attach to the ECM and connect indirectly to the actin filaments through protein assemblies of talinpaxillin-vinculin. These protein assemblies stabilise the focal adhesion structure, as well as relaying signals from the ECM to the nucleus. Integrins are heterodimer proteins composed of $\alpha$ and $\beta$ subunits. $\beta$ subunits are divided into 8 types and can associate with 16 different a chains (Hynes, 2002). The types of heterodimers formed from these complexes are dependent on the signals found on the ECM. Many ECM proteins can be involved in cell adhesion and all include domains that contain a specific amino acid sequence, each sequence is a signal for a specific integrin complex attachment site. Signals from the integrins are relayed to the nucleus by the bridging proteins and the actin fibres (Juliano and Haskill, 1993; Juliano, 2002). These signals initiate nuclear gene expression that subsequently sends the corresponding response signal. 


\section{Focal adhesion associated proteins in detail}

The focal adhesion is a structural unit that additionally directs the flow of information from outside the cell to the inside. The cell processes the relayed information and reacts according to cues it receives from its constantly changing environment. Below is a brief summary of some of the most important components involved in the focal adhesion's structural and signalling capabilities.

Integrins are transmembrane proteins that bind to peptide moieties such as Arg-Gly-Asp (RGD) that reside in the ECM, which is adsorbed to the substrate (Giancotti and Ruoslahti, 1999). The RGD moiety is present in a number of proteins such as fibronectin, vitronectin and fibrinogen, and is well known specifically to interact with a number of integrin cell receptors (Ruoslahti, 1991; 1996). On the cytoplasmic side of the plasma membrane, integrins are attached to the cytoskeleton through linker proteins. It is postulated that the cell recognises the appropriate ECM moiety by the heterodimeric conformation of the integrins (Hemler, 1998). $\alpha$-actinin can crosslink actin as well as bind to the cytoplasmic domain of the linker proteins zyxin (Honda et al., 1998) and vinculin (Crawford et al., 1992). An interaction between the signalling molecule phosphotidyl inositol-4-5 bisphosphate (PIP2) has also been reported (Shibasaki et al., 1994). Focal adhesion kinase (FAK) is a non-receptor protein tyrosine kinase, principally localised in the focal adhesion (Parsons, 2003). It is a protein, which is activated enzymatically upon engagement of the integrins with the ligands, and could be involved in regulating cell spreading, migration, and generation of an adhesion dependent anti-apoptotic signal (Ilic et al., 1997). Paxillin and tensin are downstream components of FAK signalling. Both of these proteins are localised at actin-membrane attachment sites (Turner, 2000). Information regarding the role of tensin in the focal adhesion is sparse; however, paxillin is currently the subject of extensive investigation. The main function of paxillin is as an adaptor recruiting signalling component at the cytoskeletal-membrane interface utilised in processes such as cell migration and gene expression (Brown and Turner, 2004). During signalling, tyrosine is phosphorylated in an integrin-binding dependent manner (Burridge et al., 1992). Additionally, tyrosine has the capacity to bind to vinculin and FAK - but binding to these proteins is neither necessary nor sufficient for paxillin recruitment to the focal adhesion site (Turner and Miller, 1994). However, this binding stabilises the association of paxillin with the focal adhesion. Talin is localised in adherens-type junctions with the ECM and binds in vitro to the cytoplasmic domains of integrins, vinculin, actin (Hemmings et al., 1996) and FAK (Chen et al., 1995). It is one of several proteins attaching actin filaments to the integrins, indicating that it has a key role in cell adhesion (Critchley, 2004). Studies where the expression of talin is inhibited show cells with a rounded morphology. By downregulating talin, using antisense constructs, cells demonstrate slower spreading (Albiges-Rizo et al., 1995). The fact that talin can bind to integrins and nucleate, crosslink, and bundle actin filaments further establishes its importance in the focal adhesion model. It is postulated to serve as a linker of the actin cytoskeleton to the cytoplasmic domain of the integrins.

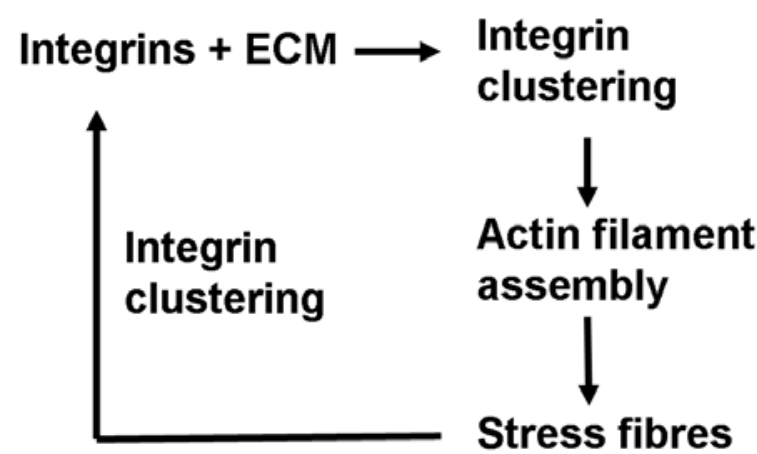

Figure 2: The positive signalling mechanism between integrins and cytoskeleton assembly.

Talin's ability to bind vinculin establishes its importance in stabilising adhesions. Vinculin is a linker protein specifically associated with focal adhesions as well as with adherens type cell-cell junctions and is present in a wide variety of cell types (Geiger, 1979). It is responsible for actin attachment to the plasma membrane, since it can interact with many of the linker proteins involved in such binding interactions. Vinculin can also bind to talin (Burridge and Mangeat, 1984), $\alpha$-actinin (Wachsstock et al., 1987), paxillin (Turner et al., 1990), actin (Gilmore and Burridge, 1996) and acidic phospholipids (Goldmann et al., 1996), as well as other vinculin molecules signifying direct interactions with proteins as well as the cell membrane (Fukami et al., 1994). The structure of vinculin is a head-tail conformation. This structure allows for multiple protein-binding sites, but these sites are hidden when the protein is in its 'curled' conformation (Winkler et al., 1996). Only when the tail extends do binding domains for other linker proteins become exposed, induced by PIP2 present in the cell membrane (Fukami et al., 1994). Zyxin is sparse, when compared to the other linker proteins. It has been proposed as a molecular scaffold, facilitating the assembly of functional complexes at sites of actin-membrane interactions (Beckerle, 1986).

\section{Signal transduction}

Cells adhere to surfaces initially by attaching to a preadsorbed protein network called the ECM or to neighbouring cells. The cells spread out and their shape (influenced by the surface topography) contributes to their phenotypic behaviour. The nature of the ECM influences major cellular programmes of growth, differentiation and apoptosis and its composition will ultimately determine which programmes will be selected. Cells "perceive" and react to the ECM by means of integrin dependent activation of intracellular signalling mediators. Integrins may transduce signals to the cytoskeleton by associating with the bridging proteins of the focal adhesion i.e talin, paxillin and vinculin. Ultimately, integrin signalling and the cytoskeleton are linked in a positive feedback mechanism inducing ECM production by the cell itself (Figure 2). Integrins can activate various protein tyrosine kinases that catalyse the 


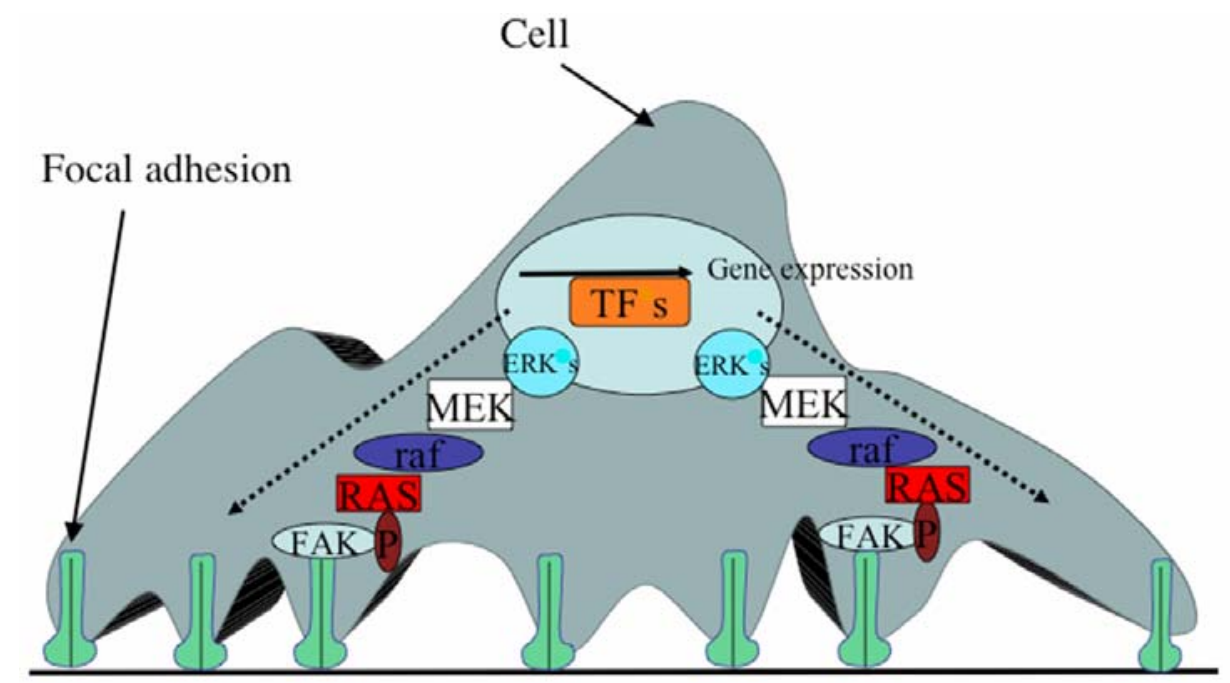

Substrate

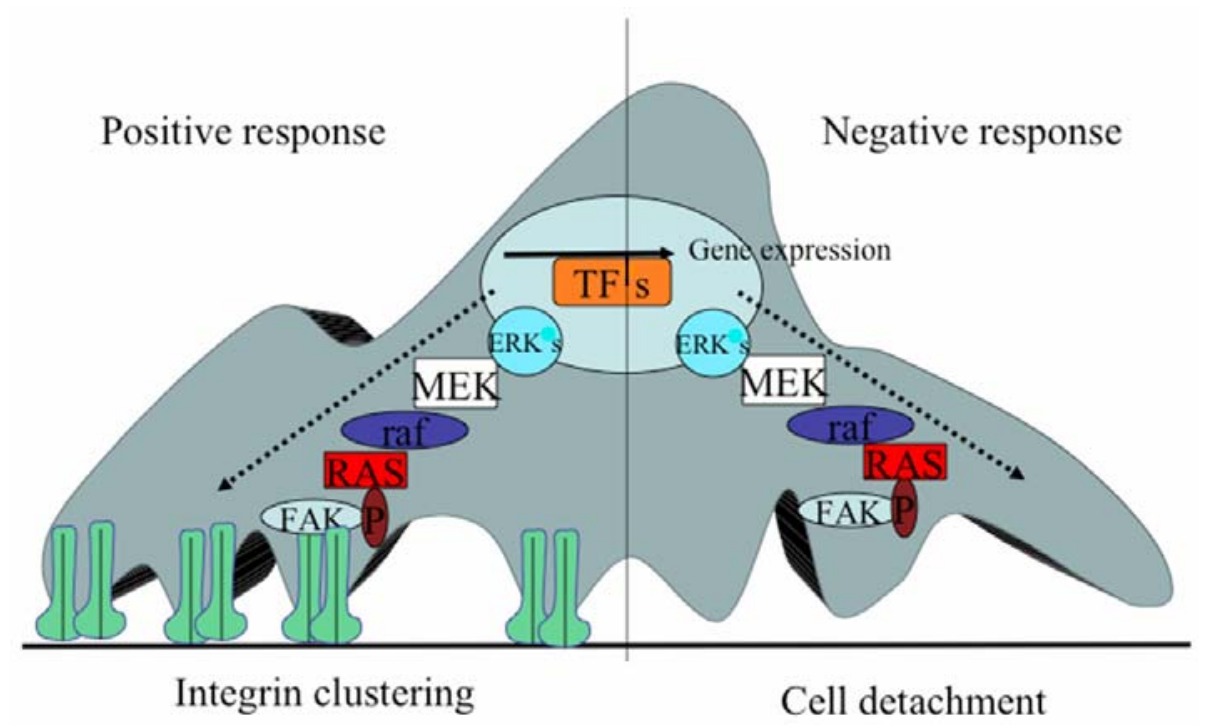

Figure 3. Representation of how integrin-mediated activation of FAK/MAPK signal tranduction pathway may regulate the cell/substrate interaction. Based on Boudreau and Jones, 1999. (a) Once the cell comes into contact with the substrate it forms focal adhesions. The integrins are thought to relay signals to the nucleus through the MAPKase (Mitogen-activated protein kinase) pathway that is a cascade of proteins abbreviated FAK $=$ Focal Adhesion Kinase, $\mathrm{P}=$ phosphorylation, $\mathrm{TFs}=$ transcription factors, RAS \& raf monomeric GTPases, ERKs = extracellular signal related kinases, MEK= MAP kinase kinase. (b) If the signals relayed by the focal adhesions to the nucleus are positive then integrin clustering occurs increasing the area of cell adhesion to the substrate, if the signals are negative then matrix metalloproteinases are released causing integrin substrate detachment decreasing the area of cell attachment. In the extreme, cases of substrate unsuitability cell detachment may occur.

phosphorylation of a number of intracellular proteins. The integrin-dependent pathways involving focal adhesion kinase (FAK) and the Src-family kinases are known in detail. The FAK pathway is activated by most integrins and also by the epitope; upon binding to the proteins of the focal adhesion a series of protein autophosphorylations occur. Ultimately, this activates the mitogen-activated protein kinase (MAPK) cascades. The consequence of activating the MAPK signal-transduction pathway is transcriptional regulation of genes - that are crucial for growth and differentiation. Cells exist in a dynamic state and they must perceive, respond and modify in response to their environment. The MAPK pathway has the potential to modulate the extent of attachment depending on the signals it receives by changing integrin-receptor expression by the "inside-out, outside-in" paradigm (Boudreau and Jones, 1999) (Figure 3). Therefore the initial cell adhesion is critical to these processes and is therefore a major determinant of a surface's cytocompatibility in the in vitro situation, and is suggested as a possible determinant of biocompatibility in the in vivo situation.

Focal adhesions contain high levels of tyrosinephosphorylated proteins, which signify their involvement as signalling molecules (Maher et al., 1985). Interestingly, 
the formation of focal adhesions is accompanied by tyrosine phosphorylation of FAK, paxillin and tensin. Autophosphorylation of FAK, for example, then creates a binding site for the SH2 (Src homology 2) domain of Src or Fyn - driving the recruitment and phosphorylation of other components (Schaller et al., 1994; Schlaepfer et al., 1994). Interference with tyrosine kinase prevents focal adhesion assembly and inhibition of normal phosphotyrosine turnover by phosphatase inhibitors. This causes a dramatic increase in the number and size of the focal adhesions (Romer et al., 1994). Tyrosine phosphorylation is therefore a critical event in focal adhesion formation. It is not yet clear whether tyrosine phosphorylation is the trigger for focal adhesion assembly or phosphorylation of focal adhesion components. Linker protein regulation is under the control of the rho-family, consisting of small G-proteins. Rho is necessary for focal adhesion formation and maintenance in ECM attached cells and is activated by extracellular ligands - such as serum factors and hormones (Hall, 1998). PIP2 production is controlled directly by Rho (Chong et al., 1994) and, as mentioned previously, the increase in PIP2 production induces a conformational change in vinculin structure exposing cryptic sites and allowing interactions with actin, $\alpha$-actinin and talin (Gilmore and Burridge, 1996). Actin polymerisation can also be regulated by Rho since PIP2 can also uncap the actin filament inducing cell contraction. Actin controls the expression of a variety of cytoskeletal proteins (Schevzov et al., 1995). Feedback regulatory mechanisms, dependent on cellular demand for a structural protein molecule can regulate its expression (Gunning et al., 1990). Rho kinase phosphorylases the regulatory light chain of myosin II. This, then stimulates cell contractility and, as a consequence, may stimulate the formation of focal adhesions and actin filaments. For additional reading see Blystone, 2004; DeMali et al., 2003; Brakebusch and Fassler 2003 and Calderwood et al., 2000. This combination of complex regulation systems ultimately controls and determines the extent of cell contact to the implant surface. Quantifying the strength or amount of cell adhesion at various timepoints is therefore useful to help determine the cyto and biocompatibility of implantable materials in vitro.

\section{Quantifying cell adhesion}

The focal adhesion is used as a means physically to attach the cell to the surface as well as relaying signals between the inside and outside of the cell. The extent of the physical attachment is dependent on the nature of the signalling the cell receives. Quantifying the extent of cell attachment seems to be a plausible way of assaying a cell's "perception" of the surface to which it is attached. The extracellular binding activity of the integrin is regulated from inside the cell and its binding to the ECM elicits signals that are transmitted into the cell. If the cell "senses" positive signals, then additional integrins are expressed and ECM proteins are synthesised. If the cell "senses" that the surface is unsuitable for adhesion then production of degrading proteases is induced, so that the cell is released from the substrate (Boudreau and Jones, 1999). For cytocompatibility testing, quantifying the amount of cell attachment should be proportional to the number of attached focal adhesion sites formed. Many methods have been used to measure the amount of cell attachment (dealt with at length by Richards et al., 1997). These approaches can be divided into two groups, mechanical and nonmechanical.

\section{Mechanical methods}

Coman (1944) first published a method to measure cellcell adhesion. This involves estimating the force necessary to bend a micropipette attached by suction to a single cell. However, this method is unsuitable for cell-substrate adhesion measurement. There are many elaborate methods, based on shear stress monitoring, devised to measure the attachment of cells to a substrate. Centrifugal force or liquid flow based methods are designed to measure the force required to remove cells from a substrate. However, cell cohesion is often measured, rather than cell substrate adhesion, during the mechanical disruption of cells on metal surfaces (Richards et al., 1995a). Atomic force microscope (AFM) cantilevers are used to measure single cell detachment from substrates. One method, attempts to measure cell adhesive strength and cell detachment surface energy (Yamamoto et al., 1998). They show variations in adhesion to different surfaces. This method could be useful to measure the individual binding strengths of integrins and their combinations to ECM, when attached to substrates. Analysis of the areas, using IRM, where cells have been detached by means of the AFM method (Yamamoto, 2001) shows that cell remainders are present - indicating that the cell is ripped off the substrate. This concurs with the observation of adhesive complex remains after applying the impingement technique (Richards et al., 1995a). Such results make attempts to quantify the strength of cell substrate adhesion using this mechanical method questionable, as these methods measure cell cohesion rather than cell adhesion.

\section{Non-mechanical methods}

Curtis (1964) demonstrates using IRM, variations in the area of cell adhesion to different substrates. These observations, confirmed by Lotz et al., (1989) show that, after the application of centrifugal force, cells with the greatest adhesion area (focal adhesions within the $10-15 \mathrm{~nm}$ surface approach range) are most resistant to detachment. One disadvantage of using IRM is that only transparent substrates can be used, therefore, for non-transparent surfaces the focal adhesion must be labelled for identification. Hunter et al., (1995) measured the area of fluorescence immunolocalisation of vinculin, in cells on different substrates. They measured the average cell spread area, as well as total focal adhesion area and then compare the ratio of focal adhesion area to the cell spread area. A direct correlation is shown between total spread area, and total vinculin area in fibroblasts. When these factors are compared for fibroblasts seeded on different substrates, significant differences are found in the focal adhesion/cell area ratios to tissue culture plastic, titanium and cobalt/ chromium/molybdenum metals, as compared to ultra-high- 
molecular-weight-polyethylene. The method is not sufficiently sensitive to compare adhesion of fibroblasts to similar metals, a limitation probably determined both by the nature of the fluorescent label (e.g bleaching) and difficulties of distinguishing labels from cytoplasmic autofluorescence within thicker less spread cells. Applying a similar approach using the scanning electron microscope (SEM) provides a greater resolution and allows direct visualisation of focal adhesions at the cell-substrate interface (independent of the cell thickness). Preferential staining of focal adhesion proteins, using osmium tetroxide, followed by embedding of the cells, removal of the original substrate, and imaging with high current low voltage backscattered electron (BSE) imaging (Richards et al., 1995b) enables the identification of focal adhesions by SEM (Richards et al., 1997). Significant differences, as quantified by digital image analysis of the stained areas, are shown between cell adhesion to Thermanox plastic and metals for different cell and metal types. However, the validity of the results is limited by large data variance, believed to be due to variations of cell adhesion within the stages of the cell cycle. With this method it is also not possible to detect the effects of minor substrate topographical differences upon cell adhesion. By applying immunocytochemical cell adhesion identification techniques it proved possible significantly to improve the sensitivity of the technique, such that differences in adhesion to more subtly differing substrates could be detected (Richards et al., 2001).

\section{Immunocytochemistry for cell adhesion quantification}

Immunocytochemistry enables the detection of an antigen, within a cell, by applying an antibody specifically directed against it. Antibodies are formed as a recognition system in higher organisms, when a foreign body or antigen is encountered in the body. The antibody recognises a unique portion of the antigen called the determinant or epitope. By augmenting the immune response it is possible artificially to produce a sufficient supply of antibodies to enable localisation studies of antigens to be carried out. Antibodies can only be identified if a detectable marker has been conjugated to them. This marker can be applied directly, either to the primary antibody or onto another secondary antibody raised against it - called indirect labelling (Polak and Van Noorden, 1997). The indirect labelling technique is the most commonly used. Antibodyantigen binding is a highly specific process; therefore this method of identification is the most reliable method for localising the protein vinculin in focal adhesion complexes. The method is limited by the type of marker conjugated to the antibody (resolution - by size of marker conjugated to antibody, resilience of marker-stability while imaging and the resolution limitations of the imaging method used to detect the marker). Sample preparation, to stabilise and preserve the epitopes within the tissue, is also crucial. Fluorescent labelling, combined with light microscopy, provides limited resolution in this context (approximate optical lateral resolution of $300 \mathrm{~nm}$ ). In addition, fluorescent labels are unstable under UV light resulting in a rapid a reduction in signal intensity, thus limiting the sensitivity of the method. Colloidal gold particles (1 to $10 \mathrm{~nm}$ diameter) provide an indelible antibody label (Faulk and Taylor, 1971) that is detectable using BSE imaging using a high-resolution field emission SEM (FESEM). BSE imaging exploits atomic number contrast available in the specimen. Gold particles provide a sharp atomic number contrast when set against biological material (Soligo et al., 1986). Improved signal to noise conditions are provided by applying the high current BSE technique (Richards et al., 1997). This provides a reliable and sensitive, high resolution technique for the detection and quantitation, by digital image analysis, of cell adhesion sites. Detection of vinculin in focal adhesion sites by imaging of small $(5 \mathrm{~nm})$ colloidal gold label, using FESEM BSE images, provides high resolution detection of such sites. However, simultaneous low magnification imaging is also necessary for measuring the total cell area with respect to the number of focal adhesion sites. In order to achieve this, the gold particles can be enlarged to detectable sizes visible at lower magnifications, by a process known as autometallography. Enlargement of the gold probe can be performed by controlled selective deposition upon it in a time-dependent manner. This process is called silver enhancement (Holgate et al., 1983; Danscher and Norgaard, 1983). However, some difficulties arise when attempting to use this method to enhance gold labelling attached to cells that are adhering to metal substrates. Owen et al.(2001) reveal the reasons for this problem and show that enlarging the small gold probes with silver is impeded, on metal substrates, by the use of osmium tetroxide containing fixatives - a necessary step in contrasting cells for electron microscopy. This problem is solved by using a gold-based enhancer, rather than a silver enhancer. It is now possible to assay the number of focal adhesions made by a cell to metal substrates with some accuracy. A remaining source of variance in the data derives from differences in the amount of cell adhesion according to cell cycle phase.

\section{Why label vinculin for focal adhesion quantification?} Identifying a focal adhesion complex is possible by labelling any protein within it. Because of the heterodimeric nature of the integrins it would be impractical to attempt labelling all combinations. Labelling components with more permanent conformations, such as the linker proteins, would be easiest but it is important to understand their role in the focal adhesion. Many focal adhesion linker proteins could be labelled. However, both dot and dash focal adhesion variants must be identified since both are involved in anchorage. Dot contacts contain only the linker proteins vinculin and $\alpha$-actinin. Dash contacts contain vinculin, $\alpha$-actinin and other linker proteins. Talin could also be present in both dot and dash contacts, although not reported, since it is recruited to newly forming focal adhesions before vinculin (Hemmings et al., 1996). The three proteins $\alpha$-actinin, talin and vinculin were possible candidates for the immunolabelling of focal adhesion complexes. The fact that $\alpha$-actinin is found associated with both cytoplasmic actin filaments and focal adhesions compromised its specificity for the purpose of 
quantifying such sites. This left only talin and vinculin. Vinculin was chosen for labelling due to the availability of commercial monoclonal antibodies against it that would bind to vinculin from several species. Such factors were considered important if the application of the quantification method was to be reproducible when applied to many cell types. However, for the correct interpretation of immunolabelling patterns the role of vinculin and its associated signalling proteins must be understood.

Vinculin is a ubiquitous protein, present in a wide variety of cell types. Within the cell, it is found in two cytoplasmic pools; the diffusible as well as the junctional membrane fraction (Meyer et al., 1997). This was an important factor to consider when designing an immunolabelling protocol. Many authors report that vinculin can react with many cytoskeletal linker proteins as well as the membrane associated signalling molecule PIP2, suggesting that vinculin can bind directly to the plasma membrane (Fukami et al., 1994; Goldmann et al., 1996). From these observations it seems that vinculin serves as a mechanical stabiliser (Alenghat et al., 2000). Transfection studies show that vinculin restores cell adhesion and normal actin organisation in adhesion deficient cells lines (Grover et al., 1987). Application of an antisense construct results in transfected cells exhibiting a rounded phenotype with fewer vinculin-positive focal adhesions with a concomitant increase in cell motility (Rodriguez Fernandez, 1993). Increasing the expression of vinculin promotes cell adhesion and reduces cell motility (Rodriguez Fernandez, 1992). In certain cellular processes the control of cell adhesion is important. For example, in conditions of quiescence, growth-arrested cells can be stimulated by platelet derived growth factor (PDGF) allowing the cells to proceed from $\mathrm{G}_{0}$ and enter the cell cycle (Herman and Pledger, 1985). During this transformation the cell must remain adherent to the substrate whilst simultaneously having the freedom to change its shape. Focal adhesions remain intact after exposure to PDGF, but vinculin specifically is removedconfirming the observations that focal adhesions are present even in vinculin deficient cell lines. Other mechanisms exist, therefore, for focal adhesion formation in the absence of vinculin. In conclusion, vinculin potentially serves as a stabilising protein in the focal adhesion, therefore the amount of vinculin present may be indicative of the motility of a cell on a given substrate. Vinculin appeared to be the most appropriate protein to target for the development of an immunocytochemistrybased cell adhesion assay method. However, it was essential to ensure that the detection of the label was restricted to that present in the focal adhesion complex (FA vinculin).

\section{Eliminating the causes of variation- the specific labelling and imaging of FA vinculin}

Vinculin present in the focal adhesion, as opposed to the diffusible cellular fraction, is resistant to Triton X-100 extraction (Niederiter et al., 1994). Treatment with this detergent is used as a means to retain only the FA vinculin. The unbound cytoplasmic fraction is removed by the Triton
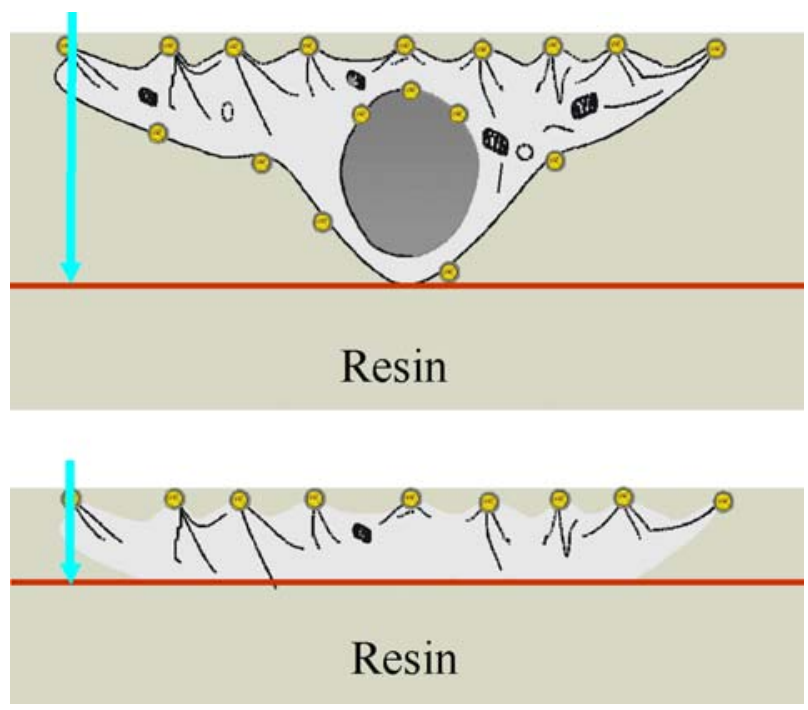

Figure 4. Diagrammatic representation of the effect of varying electron beam energy upon the depth of electron penetration and BSE emergence. The sample is immunolabelled, embedded in resin, allowing for easy removal of the substrate leaving the cell undersurface exposed in the resin. The sample is inverted and imaged using high voltage electron images to identify the whole cell (upper diagram) and low accelerating voltage to image only the immunogold labelling of focal adhesions close to the specimen surface (lower diagram).

before chemical fixation of the cell, thereby decreasing the chance of the non-specific labelling of antigen from this source (Mayer et al., 1997). Normally, Triton is used as a cell membrane permeabilising agent, to facilitate antibody penetration of the cytoplasm to enable it to reach the antigen within the cell, following fixation (Beesley, 1993). Triton extraction of the cell before labelling and fixation removes most of the unbound vinculin that is not associated with focal adhesions, as well as promoting the penetration of the primary antibody as far as the focal adhesion complex. The concept of "electron energy sectioning", using BSE imaging with an FESEM is introduced by Richards et al.(1997). The depth, within a specimen, from which an object can be detected is controllable by adjusting the incident beam energy. The beam energy is determined by the accelerating voltage set on the electron microscope gun. Embedding the cells, followed by removal of the original substrate and imaging the underneath directly by the high current low voltage BSE method can be used to eliminate any detection of background, resulting from the presence of cytoplasmic fraction vinculin remaining at the imaging stage. By imaging cells, in this way, it is possible selectively to detect labelling from the areas directly in contact with the substrate (30-50 $\mathrm{nm}$ from the surface) (Richards et al., 2001) (Figure 4). Such images are then used to carry out quantification of the cell adhesion area formed, by using an image analysis package. Image analysis enables the conversion of image measurement into quantitative data. 


\section{Eliminating the causes of variance - the cell cycle}

The cell division cycle is a fundamental process by which all cells are propagated (Howard and Pelc, 1951). The cycle is divided into two main periods, interphase and mitosis. During interphase cell growth occurs and nuclear DNA is replicated. This period is sub-divided into three main phases, $G_{1}, S$ and $G_{2}$. During mitosis the nuclear contents are divided into two, genetically identical, daughter nuclei. At the end of mitosis the whole cell is divided into two daughter cells by cytokinesis. The two daughter cells, if continuing to proliferate, then enter the $G_{1}$ phase. Other cells enter the non-proliferative $\mathrm{G}_{0}$ phase. Cross and ap Gwynn (1987) show cell flattening during S phase and then rounding-up of cells when approaching mitosis. Cell flattening corresponds to an increase in cell-substrate adhesion during S phase (Ohnishi, 1981 and Porter et al., 1973). Lotz et al. (1989) and Hunter et al. (1995) state that the flatter the cell the more focal adhesions are present. Therefore, the presence of cell cycle phase-related differences could be the reason for the large variance in cell adhesion measurement data, detected in previous studies. A non-invasive method, in which cell cycle stage identification can be combined with quantification of focal adhesion, is shown by Owen et al. (2002). This provides the ability to measure the density of focal adhesion sites, at the cell-substrate interface, on specifically identified Sphase cells. Changes in adhesion during the cell cycle have been investigated by Meredith et al. (2003). The density of focal adhesions does not appear to be different in $\mathrm{S}$ and non-S phase cells. Differences in adhesion appear to be more dependent on cell morphology. Flattened cells have more area of adhesion than those of rounded morphology. However, S-phase cells do show a more consistent adhesion density. Therefore, any change in cell adhesion that may be related to the substrate could be attributable to the nature of the substrate. The addition of specific high resolution labelling of vinculin and resolution of cell cycle phases to the quantification technique enables the revelation of significant differences between the area of cell adhesion to various materials and substrate roughnesses (Owen et al., 2002). Interestingly, one surface regarded as "very rough" produced greater data variance than the "least rough" surface, suggesting that the different surface characteristics within the rough sample itself (nano roughness, micro roughness and macro roughness) might be a reason for the variance (Owen, 2002).

Future methodology to improve method sensitivity. Variance in the cell adhesion data, attributed to the underlying differences in substrate surface roughness dimensions, decreases the significance of the experimental results. Identifying the effect of small changes in topography is important for analysing cell behaviour control but needs to be approached in a different manner. The density of cell adhesion areas appears to differ according to the local substrate roughness dimensions and is distributed in a non-homogeneous manner on the rougher surfaces. Assaying a larger sample number, to accommodate the variance in the data, should improve the sensitivity of the technique. However, applying image analysis to images of immunogold labelled cells is very time consuming. Obtaining even larger sample numbers would make this approach too cumbersome. It is possible selectively to remove free vinculin, by the application of the detergent Triton X-100 - leaving only the focal adhesion fraction of vinculin attached to the substrate. Then, remaining focal adhesion vinculin could possibly be isolated and quantified using conventional biochemical techniques. Such an approach could reduce the time consuming steps required for immunogold quantification, and increase the statistical significance of the analysis.

\section{Conclusion}

Focal adhesion quantification is a suitable method for helping to identify cytocompatible materials and surfaces. The choice of method used for quantification purposes is very important and may limit the outcome of cytocompatibility studies. Each technique has its limitations and the results should be interpreted accordingly. If a sensitive analysis is required, then the method used to identify the focal adhesions should be specific and suitable for high resolution imaging methods. The results should be related to the surface characterisation data. Combining such techniques makes this approach to quantification very labour intensive. New methods are currently being designed, based on biochemical strategies, to enable use to be made of high cell number assays. Such an approach should decrease the data variance caused by variations in substrate roughness. Reducing data variance should make it possible to discriminate between the degree of cell adhesion to more subtly different substrates and provide a clearer indication of what may comprise the most cytocompatible surface.

\section{References}

Albiges-Rizo C, Frachet P, Block MR (1995) Down regulation of talin alters cell adhesion and the processing of the alpha 5 beta 1 integrin. J Cell Sci 108: 3317-3329.

Alenghat FJ, Fabry B, Tsai KY, Goldmann WH, Ingber DE (2000) Analysis of cell mechanics in single vinculindeficient cells using a magnetic tweezer. Biochem Biophys Res Commun 277: 93-99.

Ambrose EJ (1961) The movements of fibrocytes. Exp Cell Res 8: 54-73.

Anderson JM (1993) Mechanisms of inflammation and infection with implanted devices. Cardiovasc Pathol 2: S33-S41.

Baier RE, Dutton RC (1969) Initial events in interactions of blood with a foreign surface. J Biomed Mater Res 3(1): 191-206.

Beckerle MC (1986) Identification of a new protein localised at sites of cell-substrate adhesion. J Cell Biol 103: 1679-1687.

Beesley JE (1993) Immunocytochemistry: A Practical Approach. Oxford University Press, UK.

Bershadsky AD, Tint IS, Neyfakh A, Vasiliev JM (1985) Focal contacts of normal and RSV-transformed Quail cells, Hypothesis of the transformation-induced 
deficient maturation of focal contacts. Exp Cell Res 158: 433-444.

Blystone SD (2004) Integrating an integrin: a direct route to actin. Biochim Biophys Acta 169: 47-54.

Boudreau NJ, Jones PL (1999) Extracellular matrix and integrin signalling: the shape of things to come. Biochem J 339: 481-488.

Brakebrusch C, Fassler R (2003) The integrin-actin connection, an eternal love affair. EMBO J 228: 23242333.

Brown MC, Turner CE (2004) Paxillin: adapting to change. Physiol Rev 84: 1315-1339.

Burridge K, Mangeat P (1984) An interaction between vinculin and talin. Nature 308: 744-746.

Burridge K, Fath K, Kelly T, Nuckolls G, Turner C (1988) Focal adhesions: transmembrane junctions between the extracellular matrix and the cytoskeleton. Annu Rev Cell Biol 4: 487-525.

Burridge K, Turner CE, Romer LH (1992) Tyrosine phosphorylation of paxillin and pp125FAK accompanies cell adhesion of extracellular matrix: a role in cytoskeletal assembly. J Cell Biol 119: 893-903.

Calderwood DA, Shattil SJ, Ginsberg MH (2000) Integrin and actin filaments: reciprocal regulation of cell adhesion and signalling. J Biol Chem 275: 22607-22610.

Chen H-C, Appeddu PA, Parsons JT, Hildebrand JD, Schallers MD, Guan J (1995) Interaction of focal adhesion kinase with cytoskeletal protein talin. J Biol Chem 270: 16995-16999.

Chong LD, Traynor-Kaplan A, Bokoch GM, Schwartz MA (1994) The small GTP-binding protein Rho regulates a phosphotidyl-4-phosphate 5-kinase in mammalian cells. Cell 79: 507-513.

Coman DR (1944) Adhesiveness and stickiness: Two independent properties of the cell surface. Cancer Res 21: 1436-1438.

Cooper JA (1987) Effects of cytochalasin and phalloidin on actin. J Cell Biol 105: 1473-1478.

Cornell R (1969) Cell-substrate adhesion during cell culture. An ultrastructural study. Exp Cell Res 58: 289295.

Crawford AW, Michelsen JW, Beckerele MC (1992) An interaction between zyxin and alpha-actinin. J Cell Biol 116: 1381-1393.

Critchely DR (2004) Cytoskeletal proteins talin and vinculin in integrin-mediated adhesion. Biochem Soc Trans 32: 831-836.

Cross SJ, ap Gwynn I (1987) Adhesion and the cell cycle in cultured L929 and CHO cells. Cytobios 50: 4162.

Curtis ASG (1964) The mechanism of adhesion of cells to glass. A study by interference reflection microscopy. J Cell Biol 20: 199-215.

Danscher G, Norgaard JO (1983) Light microscopic visualisation of colloidal gold on resin-embedded tissue. J Histochem Cytochem 31: 1394-1398.

Dee KC, Puelo DA, Bizios R (2002) In: An Introduction to tissue biomaterial interactions. John Wiley \& Sons New Jersey, USA. 127-147.

DeMali KA, Wennerber K, Burridge K (2003) Integrin signaling to the actin cytoskeleton. Curr Opin Cell Biol
15: $572-582$.

Dembo M, Wang YL (1999) Stresses at the cell-tosubstrate interface during locomotion of fibroblasts. Biophys J 76: 2307-2316.

Faulk WR, Taylor GM (1971) An immunocolloid method for the electron microscope. Immunochemistry $\mathbf{8}$ : 1081-1083.

Fukami K, Endo T, Imamura M, Takenawa T (1994) alpha-Actinin and vinculin are PIP2-binding proteins involved in signalling by tyrosine kinase. J Biol Chem 269: 1518-1522.

Geiger B (1979) A 130k protein from chicken gizzard. Its localization at the termini of microfilament bundles in cultured chicken cells. Cell 18: 193-205.

Giancotti FG, Ruoslahti E (1999) Integrin signalling. Science 285: 1028-1032.

Gilmore AP, Burridge K (1996) Regulation of vinculin binding to talin and actin by phosphatidyl-inositol-4-5bisphosphate. Nature 381: 531-535.

Goldman WH, Ezzell RM, Adamson ED, Niggli V, Isenberg G (1996) Vinculin, Talin and focal adhesions. J Musc Res and Cell Mot 17: 1-5.

Grover A, Rosentraus MJ, Sterman B, Snook ME, Adamson ED (1987) An adhesion-defective variant of F9 embryonal carcinoma cells fails to differentiate into visceral endoderm. Dev Biol 120: 1-11.

Gunning P, Gordon M, Wade R, Gahlmann R, Lin CS, Hardemann E (1990) Differential control of tropomyosin mRNA levels during myogenesis suggests the existence of an isoform competition-autoregulatory compensation control mechanism. Dev Biol 138: 443-453.

Hall A (1998) Rho GTPases and the actin cytoskeleton. Science 279: 509-514.

Hemler ME (1998) Integrin associated proteins. Curr Opin Cell Biol 10: 578-585.

Hemmings L, Rees DJ, Ohanian V, Bolton SJ, Gilmore AP, Patel B, Priddle H, Trevithick JE, Hynes RO, Critchley DR (1996) Talin contains three actin-binding sites each of which is adjacent to a vinculin-binding site. J Cell Sci 109: 2715-2726.

Herman B, Pledger WJ (1985) Platelet-derived growth factor-induced alterations in vinculin and actin distribution in BALB/c-3T3 cells. J Cell Biol 100: 1031-1040.

Holgate CS, Jackson P, Cowen PN, Bird CC (1983) Immunogold-silver staining: new method of immunostaining with enhanced sensitivity. J Histochem Cytochem 31: 938-944.

Honda K, Yamada T, Endo R, Ino Y, Gotoh M, Tsuda H, Yamada Y, Chiba H, Hirohashi S (1998) Actinin-4, a novel actin-bundling protein associated with cell motility and cancer invasion. J Cell Biol 140: 1383-1393.

Howard A, Pelc SR (1951) Nuclear incorporation of $\mathrm{P}^{32}$ as demonstrated by autoradiographs. Exp Cell Res 2: 178-187.

Hunter A, Archer CW, Walker PS, Blunn GW (1995) Attachment and proliferation of osteoblasts and fibroblasts on biomaterials for orthopaedic use. Biomaterials 16: 287 295.

Hynes RO (1987) Integrins: a familiy of cell surface receptors. Cell 48: 549-554.

Hynes RO (2002) Integrins: bidirectional, allosteric 
signaling machines. Cell 110: 673-687.

Ilic D, Damsky CH, Yamamoto T (1997) Focal adhesion kinase: at the crossroads of signal transduction. J Cell Sci 110: 401-407.

Izzard CS, Lochner LR (1976) Cell to substrate contacts in living fibroblasts: an interference reflexion study with an evaluation of the technique. J Cell Sci 21: 129-159.

Juilano RL (2002) Signal transduction by cell adhesion receptors and the cytoskeleton function of integrin, cadherin selectin and immunoglobulin superfamily members. Ann Rev Pharmacol Toxicol 42: 283-323.

Juliano RL, Haskill S (1993) Signal transduction from the extracellular matrix. J Cell Biol 120: 577-585.

Lotz MM, Burdsal CA, Erickson HP, McClay DR (1989) Cell adhesion to fibronectin and tenascin: Quantitative measurements of initial binding and subsequent strengthening response. J Cell Biol 109: 17951805.

Maher PA, Pasquale EB, Wang JY, Singer SJ (1985) Phosphotyrosine-containing proteins are concentrated in focal adhesions and intercellular junctions in normal cells. Proc Natl Acad Sci U S A 82: 6576-6580.

Meredith DO, Owen GRh, ap Gwynn I, Richards RG (2003) Variation in cell-substratum adhesion in relation to cell cycle phases. Exp Cell Res 29: 58-67.

Meyer U, Meyer T, Jones DB (1997) No mechanical role for vinculin in strain transduction in primary bovine osteoblasts. Biochem Cell Biol 75: 81-87.

Niederiter M, Gimona M, Streichsbier F, Celis JE, Small JV (1994) Complex protein composition of isolated focal adhesions: A two-dimensional gel and database analysis. Electrophoresis 15: 511-519.

Ohnishi R (1981) Dynamics of cultured L cells as studied by cinematography and scanning electron microscopy. Biomed Res 2: 1-12.

Owen GRh (2002) Osteoblast adhesion and ECM mineralization in implant osteointegration. $\mathrm{PhD}$ thesis, University of Wales.

Owen GRh, Meredith DO, ap Gwynn I, Richards RG (2001) Enhancement of immunogold-labelled focal adhesion sites in fibroblasts cultured on metal substrates: Problems and solutions. Cell Biol Int 25: 1251-1259.

Owen GRh, Meredith DO, ap Gwynn I, Richards RG (2002) Simultaneously identifying S-phase labelled cells and immunogold-labelling of vinculin in focal adhesion sites. J Microsc 207: 27-36.

Parsons JT (2003) Focal Adhesion kinase: the first ten years. J Cell Sci 116: 1409-1416.

Pollack JM, Van Noorden S (1997) Introduction to Immunocytochemistry. $2^{\text {nd }}$ Edition. Bios Scientific Publishers, Oxford, UK.

Porter KR, Prescott D, Frye J (1973) Changes in surface morphology of Chinese hamster ovary cells during the cell cycle. J Cell Biol 57: 815-836.

Richards RG, ap Gwynn I (1995b) Backscattered electron imaging of the undersurface of resin-embedded cells by field emission scanning electron microscopy. J Microsc 177: 43-52.

Richards RG, ap Gwynn I, Bundy KJ, Rahn BA (1995a) Microjet impingement followed by scanning electron microscopy as a qualitative technique to compare cellular adhesion to various biomaterials. Cell Biol Int 19: 10151024.

Richards RG, Owen GRh, Rahn BA, ap Gwynn I (1997) A quantitative method of measuring cell-substrate adhesion areas. Cell Mater 7: 15-30.

Richards RG, Stiffanic M, Owen GRh, Riehle M, ap Gwynn I, Curtis ASG (2001) Immunogold labelling of fibroblast focal adhesion sites visualised in fixed material using scanning electron microscopy and in vivo, using internal reflection microscopy. Cell Biol Int 25: 1237-1249.

Rodriguez Fernandez JL, Geiger B, Salomon D, BenZe'ev A (1992) Overexpression of vinculin suppresses cell motility in BALB/c 3T3 cells. Cell Motil Cytoskeleton 22: 127-134.

Rodriguez Fernandez JL, Geiger B, Salomon D, BenZe'ev A (1993) Suppression of vinculin expression by antisense transfection confers changes in cell morphology, motility, and anchorage-dependent growth of 3 T3 cells. J Cell Biol 122: 1285-1294.

Romer LH, McLean N, Turner CE, Burridge K (1994) Tyrosine kinase activity, cytoskeletal organization, and motility in human vascular endothelial cells. Mol Biol Cell 5: 349-361.

Rouslahti E (1996) RGD and other recognition sequences for integrins. Annu Rev Cell Dev Biol 12: $697-$ 715 .

Ruoslahti E (1991) Integrins as receptors for extracellular matrix. In: Hay ED, ed. Cell biology of extracellular matrix. New York - Plenum Press 343-363.

Schaller MD, Hildebrand JD, Shannon JD, Fox JW, Vines RR, Parsons JT (1994) Autophosphorylation of the focal adhesion kinase, pp125FAK, directs SH2-dependent binding of pp60src. Mol Cell Biol 14: 1680-1688.

Schevzov G, Lloyd C, Gunning P (1995) Impact of altered actin gene expression on vinculin, talin, cell spreading and motility. DNA Cell Biol 14: 689-700.

Schlaepfer DD, Hanks SK, Hunter T, van der Geer P (1994) Integrin-mediated signal transduction linked to Ras pathway by GRB2 binding of focal adhesion kinase. Nature 372: 786-791.

Shibasaki F, Fukami K, Fukui Y, Talenawa T (1994) Phosphotidylinositol 3-kinase bionds to alpha-actinin through the p85 subunit. Biochem J 302: 551-557.

Soligo D, de Harven E, Nava MT, Lambertenghi Dehlers G (1986) Immunocytochemistry with backscattered electrons. In: Mueller M, Becker RP, Boyde A, Wolasewick JJ, eds. Science of biological specimen preparation. pp.289-297. SEM Inc, Illinois, USA.

Turner CE (2000) Paxillin and focal adhesion signalling. Nat Cell Biol 2: E231-236.

Turner CE, Miller JT (1994) Primary sequence of paxillin contains putative $\mathrm{SH} 2$ and $\mathrm{SH} 3$ domain binding motifs and multiple LIM domains: identification of a vinculin and pp125Fak-binding region. J Cell Sci 107: 1583-1591.

Turner CE, Glenney JR Jr, Burridge K (1990) Paxillin: a new vinculin-binding protein present in focal adhesions. J Cell Biol 111: 1059-1068.

Wachsstock DH, Wilkins JA, Lin S (1987) Specific interaction of vinculin with $\alpha$-actinin. Biochem Biophys Res Commun 146: 554-560. 
Wilson CJ, Clegg RE, Leavesley DI, Pearcy MJ (2005) Mediation of biomaterial-cell interactions by adsorbed proteins: a review. Tissue Eng 11: 1-18.

Winkler J, Linsdorf H, Jockusch BM (1996) The ultrasturcture of chicken gizzard vinculin as visualised by high-resolution electron microscopy. J Struct Biol 116: 270-277.

Yamamoto A (2001) Biomechanics of Cell-Material Adhesion. Eur Cell Mat 2 (Suppl 1): 11-12.

Yamamoto A, Mishima S, Maruyama N, Sumita M (1998) A new technique for direct measurement of the shear force necessary to detach a cell from a material. Biomaterials 19: 871-879.

\section{Discussion with Reviewers}

P Bongrand: Is it possible to give a rough estimate of the lateral resolution achieved with the authors' technique?

Authors: The theoretical lateral resolution of any SEM is dependent on the beam diameter and the accelerating voltage. The lateral resolution of a cold emitter FESEM, used for this purpose was $0.5 \mathrm{~nm}$ at $30 \mathrm{kV}$, decreasing to $2.5 \mathrm{~nm}$ at $1 \mathrm{kV}$. Lateral resolution is limited more by the length of the immunological reagents linking the antigens to the dense marker $(\sim 15 \mathrm{~nm})$, than to the resolution of the SEM.

P Bongrand: Is it conceivable to design different kinds of colloidal particles to achieve something comparable to fluorescent co-localisation?

Authors: Is it feasible to use gold for co-localisation studies, since colloidal gold particles of different sizes can be conjugated to antibodies. The range of gold colloids commercially available is from 0.8 to $50 \mathrm{~nm}$ in diameter with 5, 10 and 20nm most commonly used. An advantage of using a gold particle with a larger diameter is that it is easier to detect, but has the disadvantage of lower labelling efficiency due to steric hindrance. Bearing in mind the problem of using the variety of gold particle sizes available it could be feasible to use particles within this size range. If a high resolution microscope is used with a resolving power capable of distinguishing different gold particle sizes then multiple labelling would be feasible.

P Bongrand: Isn't it more difficult to make colloidal particles enter permeabilised cells than fluorescent antibodies?

Authors: In theory the entry of fluorescently labelled antibodies into a permeabilsed cell is facilitated by their small size and the lack of charge on the fluorescent probe. Nowadays fluorescent markers are often increased in number upon the antibody, to increase signal emission, which may bring their combined dimensions closer to that of the smaller immunogold markers. However, the fluorescent markers are often very susceptible to solvents used in dehydration, embedding etc. (unlike the gold ones). For these studies we determined the most suitable size of gold $(5 \mathrm{~nm})$ by taking into consideration the penetration rate and labelling efficiency. To ease antibody access to the focal adhesions, we removed the unnecessary portions of the cell, using a detergent that does not remove or affect the vinculin epitope within the focal adhesion ensemble. Using this methodology and utilising the $5 \mathrm{~nm}$ gold label provided a very effective way of labelling the vinculin on the cell adhesion sites.

P Bongrand: When using $5 \mathrm{~nm}$ particles is detection efficiency $100 \%$ and is there a possibility of detection artefacts?

Authors: The labelling method has been designed to only detect vinculin in the focal adhesion. To understand the complexity of the labelling procedure fully, it is necessary to know the following:

The methodology utilised in this study was designed specifically to detect vinculin present only in the focal adhesion. Unbound vinculin (not present in the focal adhesions) was extracted specifically using the detergent Triton X-100, without affecting the bound vinculin. During this step the cell membrane was also permeabilised facilitating antibody penetration. The cell remains only contained the cytoskeletal components which would limit background labelling. Primary monoclonal antibodies were used to detect the focal adhesion vinculin. Monoclonal antibodies were used because they bind to only one epitope on the vinculin antigen. Polyclonal antibodies conjugated to $5 \mathrm{~nm}$ gold were used as the secondary antibodies. Polyclonal antibodies bind to multiple epitopes on the primary antibody and are a way of enhancing the detection of primary antibody antigen interaction. As a precautionary measure any non-specific binding sites were neutralised using blocking agents such as BSA and Tween 20. The technique also included an additional blocking step before the secondary antibody, incubation with goat serum (the species used to produce the secondary antibody), again limiting the background labelling that might occur. Focal adhesion imaging depth is limited to $30 \mathrm{~nm}$ of the cell undersurface. This range has been shown to be specific for the focal adhesion by IRM. No immunolabelling method has a $100 \%$ detection efficiency, due to all the stages where damage to some epitopes can occur during the actual labelling or fixation procedure. However, it should be made clear that out intention was not to quantify the number of focal adhesions but the total area of focal adhesions. With this in mind our technique is rather too specific for its purpose but as smaller topographical patterns are applied to implant materials, the application of such a sensitive technique will be more beneficial.

ASG Curtis: please comment upon the merits and demerits of using fluorescence and other optical methods on opaque substrates.

Authors: Autofluorescence is one major problem with fluorescence on numerous surfaces (especially polymers), which makes quantification more difficult. The limitations of 'one-plane' conventional fluorescence microscopy would make it difficult to resolve interaction on roughened surface for two reasons. The first is the obvious limitation of the microscope in imaging 'multiple-planes' associated with rough surfaces. The second it that generally cells do 
not spread on rougher substrates, therefore the general fluorescence of the cytoplasm interferes with the specific signal from the fluorochrome. It is true that image deconvolution methods, or confocal microscopy, could be used to reduce such problems. However, both fluorochrome bleaching and the label's poor resistance to solvents during preparation procedures would hinder any attempts at quantification. The capacity to apply multiple labelling is one of the main advantages of fluorescence microscopy. One important aspect is that imaging the focal adhesion sites on a surface with large microtopography roughnesses requires a microscope with a large depth of field. The SEM has a particularly large depth of field compared to any light microscope.

ASG Curtis: A main matter of interest in cytocompatibility studies is whether cells will form adhesions with the material. What happens after this even if they do permanently or fall off later is interesting but not so important. Please comment?

Authors: Cells are thought initially to adhere to the implant material by means of Van der Waal and electrostatic interactions. Adhesion to the implant at this stage is nonspecific, since charges on the cell surface will induce cell binding with the proteins upon the implant surface. This charge-based adhesion occurs within seconds after initial contact. When the integrin machinery is activated this is when the specificity of the adhesion becomes apparent and is dependent mainly on the implant/ECM surface energy. The surface energy of the implant is believed to induce pre-adsorption of proteins onto that surface. The proteins that are adsorbed on the surface are thought to 'inform' the cell what lies beneath, providing the inside-out outsidein signalling, providing information as to whether the surface is suitable for attachment - in preparation of proliferation. Permanent adhesion of the cell to the implant material (coated with ECM) over a long period of time is very important in the clinical sense. When a medical device is implanted into the body a series of processes are activated to isolate the foreign body. Essentially this involves encapsulating the foreign body by tissue. Cell proliferation requires that the cell adheres permanently throughout the cell division cycle. If cell detachment occurs then capsule formation does not occur and is preceded by acute inflammation resulting in a liquid filled void. This 'dead space', containing the waste products of the attack, is an ideal environment for bacteria and can cause infection.

ASG Curtis: Can you comment upon the rate of formation of adhesions?

Authors: With regards to quantifying cell adhesion from the rate of formation of adhesions this is a particularly difficult procedure because it is complicated by the different types of interactions that are being used by the cell to adhere to the substrate initially. Once it has adhered, the inside-out - outside-in signalling between the nucleus and the focal adhesion balances the extent of attachment depending on the substrate suitability. The technique we report used a time-frame of 24 or $48 \mathrm{~h}$ as time points for quantifying the area of adhesion. These timings allowed the cell to go through one cell cycle (which is also a very sensitive indicator of substrate suitability) and gives the cell time to recover from the effects of cell sub-culturing.

ASG Curtis: It is probably incorrect that rough topographies are widely associated with poor spreading and certainly not in the case of groove ridge topography but how are the adhesive contacts with the edges of the grooves best investigated since there is considerable depth of structure in most cases?

Authors: Cells adhere to substrates at different levels of topographical dimensions, termed 'windows of topography'. It has been widely documented that cells become well spread on substrates with smooth surfaces but spreading is rather limited on micro-rough surfaces. This response has been observed to be dependent on the particular 'window of roughness' experienced by the cell, governed primarily by the topography within the range of the focal adhesion dimensions. Fabricated groove ridge topography window of roughness can be deemed rough from a topographical stand point, but is essentially smooth at the scale of focal adhesions (apart from at the groove/ ridge boundaries). This discrepancy may be due to this particular factor being overlooked. Imaging adhesive contacts on a grooved-ridge topography is very difficult by light microscopy based imaging methods, but can easily be visualised with an SEM. The limiting factor of other methods, compared to the SEM, is the depth of field (the depth that is in focus in the specimen). Depth of field, in an SEM, is affected by the distance the specimen is situated from the final condenser lens (the working distance) and is controlled by the aperture angle. The aperture angle can be varied by moving the specimen physically, with respect to the condenser lens, or by using condenser apertures with smaller diameters. The high current backscattered electron technique, used for imaging the immunogold label in this method, has been optimised so that the depth of field is of the order of microns. This is sufficient for this particular application and would be suitable for imaging adhesive contacts on substrates with groove-ridge nano and micro topography. 